\title{
Global Behavior of a Computer Virus Propagation Model on Multilayer Networks
}

\author{
Chunming Zhang $\mathbb{D}$ \\ School of Information Engineering, Guangdong Medical University, Dongguan 523808, China \\ Correspondence should be addressed to Chunming Zhang; chunfei2002@163.com
}

Received 10 October 2017; Revised 2 February 2018; Accepted 4 March 2018; Published 12 April 2018

Academic Editor: Vasileios A. Karyotis

Copyright (C) 2018 Chunming Zhang. This is an open access article distributed under the Creative Commons Attribution License, which permits unrestricted use, distribution, and reproduction in any medium, provided the original work is properly cited.

\begin{abstract}
This paper presents a new linear computer viruses propagation model on multilayer networks to explore the mechanism of computer virus propagation. Theoretical analysis demonstrates that the maximum eigenvalue of the sum of all the subnetworks is a vital factor in determining the viral prevalence. And then, a new sufficient condition for the global stability of virus-free equilibrium has been obtained. The persistence of computer virus propagation system has also been proved. Eventually, some numerical simulation results verify the main conclusions of the theoretical analysis.
\end{abstract}

\section{Introduction}

In 1987, the first computer viruses propagation model was proposed by Cohen [1]. Since then, numbers of typical computer viruses propagation models had been proposed, for instance, susceptible-infected-susceptible (SIS) models [2], susceptible-infected-removed (SIR) models [3, 4], susceptible-infected-recovered-susceptible (SIRS) models [5], susceptible-exposed-infected-removed-susceptible (SEIRS) models [6], susceptible-infected-patched-susceptible (SIPS) models [7], and susceptible-infected-external-susceptible (SIES) [8]. However, some of these models simply ignore the fact that the dominating majority of computer viruses have a quite long propagation period before breaking out which can vividly express the node with high infectious ability, while some other models assume that, during its latency, an infected computer just infected recently that has low infectious ability compared with breaking out nodes, also known as an $E$ computer, has no infectivity. This, however, is inconsistent with the fact that, in general, an infected computer does possess infectivity [9]. To overcome these deficiencies, Yang et al. proposed a novel computer virus propagation model, called Susceptible-Latent-Breaking-Susceptible (SLBS) model, in which all the computers connected to the Internet are divided into three groups: virus-free computers, known as susceptive computer ( $S$ computer), infected computers that are latent ( $L$ computer), and infected computers in which the viruses are breaking out that means the computer with a high infectious level ( $B$ computer). One remarkable distinction between the SLBS model and the classical SEIS model is that a latent computer possesses infecting capability [9].

While the mechanism of computer virus propagation on networks is an important research area, lots of networkbased computer viruses propagation models ranging from susceptible-infected (SI) models $[2,10]$ and SIS models [3$5,8,11-14]$ to SIR models $[5,6,13,15,16]$ and SLBS models [14] and SIPS models [7] have also been inspected. However, these studies mainly focus on single layer networks [11-13, 15, 1724]. In reality, computer viruses can spread not only through single layer networks but also through multilayer networks; for example, mobile phone viruses (a type of computer virus) can utilize $3 \mathrm{G}$ network, $4 \mathrm{G}$ network, Wi-Fi network, and even Bluetooth network as their communication network.

On the other hand, from the point of view of research methods, Markov chain method, which is proposed by Van Mieghem et al. [10, 25], can exactly describe computer viruses propagation process with constant transition rates between compartments on any networks. Nevertheless, this method is complex in mathematical analysis. For the purpose of overcoming this deficiency, several approximate methods of researching computer viruses propagation on networks are also proposed in recent years. For example, based on 
the assumption that the dynamic state of every node is statistically independent of the state of its nearest neighbors, Wang et al. [26], Youssef and Scoglio [27], and Yang et al. [14] proposed the so-called Individual-based mean-field theory (IBMF); based on the assumption that all nodes of degree $k$ are statistically equivalent, Pastor-Satorras and Vespignani $[28,29]$ and Barthélemy et al. [30] proposed the so-called degree-based mean-field theory (DBMF), and so on.

For the purpose of more accurately understanding the propagation mechanism of computer viruses on multilayer networks, in this paper, we propose a novel SLBS computer virus propagation model on multilayer network. Highlights of this paper are as follows.

(1) Based on the assumption of multilayer network, by applying the IBMF to the existing SLBS model, a highdimensional computer virus propagation dynamic model, which is known as the individual-based SLBS model, is formulated. This model forms the foundation of this work. To our knowledge, there is no report about the spread of computer viruses on multilayer networks.

(2) To find out the influence of multilayer networks topology on computer virus spreading, by means of mathematical analysis, I find out that the propagation threshold is the maximum eigenvalue of the sum of all the subnetworks on multilayer networks. Then, the global stability of the virus-free equilibrium has been analyzed. The persistence of system has been proved. Extensive experiments confirmed the conclusions of the mathematical analysis.

The subsequent materials of this paper are organized as follows. In Section 2, we present the multilayer networks and computer viruses propagation model in detail; and then in Section 3 the model is analyzed comprehensively; numerical simulation results are given in Section 4; eventually, in Section 5, we outline this work.

\section{Assumptions and Modeling}

For the purpose of describing the model in detail, the following notations are proposed:

(i) $G=(V, E)$ : the multilayer network, which consists of $n$ subnetworks

(ii) $G_{s}=\left(V_{s}, E_{s}\right)(s=1,2, \ldots, n)$ : the sth layer subnetwork; each subnetwork $G_{s}$ has $N$ nodes

(iii) $V_{s}$ : the set of nodes contained in $G_{s}$

(iv) $E_{s}$ : the set of edges contained in $G_{s}$

(v) $a_{i j}^{s}$ : the link from node $i$ to node $j$ in $G_{s}, a_{i j}^{s} \in\{0,1\}$

(vi) $A_{s}=\left[a_{i j}^{s}\right]_{N \times N}$ : the corresponding parameterized adjacency matrix of graph $G_{s}$.

In addition, a dynamic switching network $G=(V, E)$ also must satisfy the following conditions:

(I) $V=V_{1}=V_{2}=\cdots V_{n}$.

(II) $E=\bigcup_{s=1}^{n} E_{s}$ and $E_{s_{1}} \cap E_{s_{2}}=\varnothing$ for all $s_{1} \neq s_{2}$.

Condition (I) presents that nodes in all subnetworks are identical. Condition (II) shows that the edges of any two subnetworks are different.

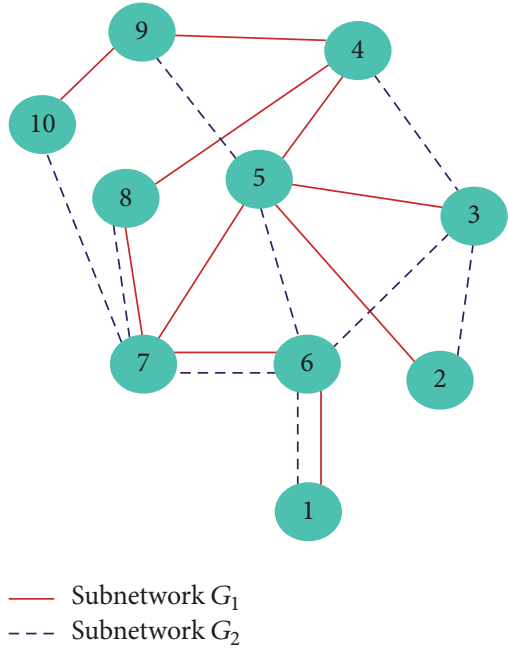

FIGURE 1: Multilayer network.

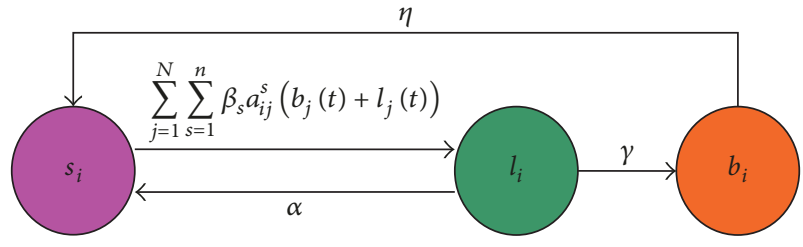

FIGURE 2: State diagram of SLBS model in multilayer networks.

A simple example of a multilayer network is shown in Figure 1 .

As discussed above in the paper, the traditional SLBS model can divide the node into three parts: susceptive node ( $S$ node) used to be known as health node and uninfected node, latent node ( $L$ node) representing the node which is infected but only showing a low infectious ability, and breaking out node ( $B$ node). Compared with $L$ node, $B$ node has significant high infectious power. Let $\xi_{i}(t)=0(1,2)$ stand for the state of the node which is susceptible (latent, breaking out) at time $t$. Then the state of the multilayer network at time $t$ can be expressed as follows:

$$
\xi(t)=\left(\xi_{1}(t), \ldots, \xi_{n}(t)\right) \in\{0,1,2\}^{n} .
$$

Let $s_{i}(t)\left(l_{i}(t), b_{i}(t)\right)$ represent the probability that node $i$ is $S$ node ( $L$ node, $B$ node) at time $t$ :

$$
\begin{aligned}
& s_{i}(t)=\operatorname{Pr}\left(\xi_{i}(t)=0\right), \\
& l_{i}(t)=\operatorname{Pr}\left(\xi_{i}(t)=1\right), \\
& b_{i}(t)=\operatorname{Pr}\left(\xi_{i}(t)=2\right) .
\end{aligned}
$$

Then, the following assumptions are given (see Figure 2): (H1) In the sth layer subnetwork $G_{s}$, the probability that a susceptible node $i$ is infected by a viral (including latent and breaking out) neighbor $j$ is $\beta_{s} a_{i j}^{s}\left(b_{j}(t)+l_{j}(t)\right)$, where $\beta_{s}$ denotes the infection rate in the sth layer subnetwork $G_{s}$. The probability that a susceptible node $i$ is infected by a viral 
neighbor $j$ in all layer subnetworks is $\sum_{s=1}^{n} \beta_{s} a_{i j}^{s}\left(b_{j}(t)+l_{j}(t)\right)$. Then, the probability that a susceptible node $i$ is infected by all viral neighbors in all subnetworks is $\sum_{j=1}^{N} \sum_{s=1}^{n} \beta_{s} a_{i j}^{s}\left(b_{j}(t)+\right.$ $\left.l_{j}(t)\right)$.

(H2) The probability that a latent node becomes a breaking out node is $\gamma$.

(H3) The probability that a breaking out node becomes a susceptible node is $\eta$.

(H4) The probability that a latent node becomes a susceptible node is $\alpha$.

Let $\Delta t$ denote a short time interval. According to the total probability theorem, several formulas can be given from the above assumptions as follows (see Figure 2):

$$
\begin{aligned}
s_{i}(t+\Delta t)= & s_{i}(t) \operatorname{Pr}\left(\xi_{i}(t+\Delta t)=0 \mid \xi_{i}(t)=0\right) \\
& +l_{i}(t) \operatorname{Pr}\left(\xi_{i}(t+\Delta t)=0 \mid \xi_{i}(t)=1\right) \\
& +b_{i}(t) \operatorname{Pr}\left(\xi_{i}(t+\Delta t)=0 \mid \xi_{i}(t)=2\right), \\
l_{i}(t+\Delta t)= & s_{i}(t) \operatorname{Pr}\left(\xi_{i}(t+\Delta t)=1 \mid \xi_{i}(t)=0\right) \\
& +l_{i}(t) \operatorname{Pr}\left(\xi_{i}(t+\Delta t)=1 \mid \xi_{i}(t)=1\right) \\
& +b_{i}(t) \operatorname{Pr}\left(\xi_{i}(t+\Delta t)=1 \mid \xi_{i}(t)=2\right), \\
b_{i}(t+\Delta t)= & s_{i}(t) \operatorname{Pr}\left(\xi_{i}(t+\Delta t)=2 \mid \xi_{i}(t)=0\right) \\
& +l_{i}(t) \operatorname{Pr}\left(\xi_{i}(t+\Delta t)=2 \mid \xi_{i}(t)=1\right) \\
& +b_{i}(t) \operatorname{Pr}\left(\xi_{i}(t+\Delta t)=2 \mid \xi_{i}(t)=2\right) .
\end{aligned}
$$

Here $o(\Delta t)$ represents time $t$ higher-order infinitesimal. According to (H1)-(H4) and (3), we can derive the following equations:

$$
\begin{aligned}
& \operatorname{Pr}\left(\xi_{i}(t+\Delta t)=0 \mid \xi_{i}(t)=0\right) \\
& =1-\left(\sum_{j=1 s=1}^{N} \sum_{s}^{n} \beta_{s} a_{i j}^{s}\left(b_{j}(t)+l_{j}(t)\right)\right) \Delta t+o(\Delta t), \\
& \operatorname{Pr}\left(\xi_{i}(t+\Delta t)=1 \mid \xi_{i}(t)=0\right) \\
& \quad=\left(\sum_{j=1 s=1}^{N} \sum_{s}^{n} a_{i j}^{s}\left(b_{j}(t)+l_{j}(t)\right)\right) \Delta t+o(\Delta t), \\
& \operatorname{Pr}\left(\xi_{i}(t+\Delta t)=2 \mid \xi_{i}(t)=0\right)=o(\Delta t), \\
& \operatorname{Pr}\left(\xi_{i}(t+\Delta t)=0 \mid \xi_{i}(t)=1\right)=\alpha \Delta t+o(\Delta t), \\
& \operatorname{Pr}\left(\xi_{i}(t+\Delta t)=1 \mid \xi_{i}(t)=1\right) \\
& \quad=1-\alpha \Delta t-\gamma \Delta t+o(\Delta t), \\
& \operatorname{Pr}\left(\xi_{i}(t+\Delta t)=2 \mid \xi_{i}(t)=1\right)=\gamma \Delta t+o(\Delta t), \\
& \operatorname{Pr}\left(\xi_{i}(t+\Delta t)=0 \mid \xi_{i}(t)=2\right)=\eta \Delta t+o(\Delta t), \\
& \operatorname{Pr}\left(\xi_{i}(t+\Delta t)=1 \mid \xi_{i}(t)=2\right)=o(\Delta t), \\
& \operatorname{Pr}\left(\xi_{i}(t+\Delta t)=2 \mid \xi_{i}(t)=2\right)=1-\eta \Delta t+o(\Delta t) .
\end{aligned}
$$

Substituting (4) into the above formulas and letting $\Delta t \rightarrow$ 0 , we deduce the $3 \mathrm{~N}$-dimensional differential system as follows:

$$
\begin{aligned}
\frac{d s_{i}(t)}{d t}= & -\left(\sum_{j=1}^{N} \sum_{s=1}^{n} \beta_{s} a_{i j}^{s}\left(b_{j}(t)+l_{j}(t)\right)\right) s_{i}(t)+\alpha l_{i}(t) \\
& +\eta b_{i}(t), \\
\frac{d l_{i}(t)}{d t}= & \left(\sum_{j=1}^{N} \sum_{s=1}^{n} \beta_{s} a_{i j}^{s}\left(b_{j}(t)+l_{j}(t)\right)\right) s_{i}(t)-\alpha l_{i}(t) \\
& -\gamma l_{i}(t), \\
\frac{d b_{i}(t)}{d t}= & \gamma l_{i}(t)-\eta b_{i}(t) .
\end{aligned}
$$

Because $s_{i}(t)+l_{i}(t)+b_{i}(t) \equiv 1, s_{i}(t)$ can be expressed by the following equation: $s_{i}(t)=1-l_{i}(t)-b_{i}(t)$. Then, the following $2 \mathrm{~N}$-dimensional subsystem can be derived:

$$
\begin{aligned}
& \frac{d l_{i}(t)}{d t} \\
& =\left(\sum_{j=1}^{N} \sum_{s=1}^{n} \beta_{s} a_{i j}^{s}\left(b_{j}(t)+l_{j}(t)\right)\right)\left(1-l_{i}(t)-b_{i}(t)\right) \\
& \quad-\alpha l_{i}(t)-\gamma l_{i}(t) \\
& \frac{d b_{i}(t)}{d t}=\gamma l_{i}(t)-\eta b_{i}(t),
\end{aligned}
$$

$$
1 \leq i \leq N
$$

Without loss of generality, we introduce an infection matrix $H=\left(h_{i j}\right) \in R^{N \times N}$, where $h_{i j}=\beta_{1} a_{i j}^{1}+\beta_{2} a_{i j}^{2}+\cdots+$ $\beta_{n} a_{i j}^{n}=\sum_{s=1}^{n} \beta_{s} a_{i j}^{s}$ (or $H=\beta_{1} A_{1}+\beta_{2} A_{2}+\cdots+\beta_{n} A_{n}$ ). We assume $\lambda_{\max }$ represents the maximum eigenvalue of matrix $H$.

Then, system (6) can be expressed as follows:

$$
\begin{aligned}
\frac{d l_{i}(t)}{d t}= & \left(\sum_{j=1}^{N} h_{i j}\left(b_{j}(t)+l_{j}(t)\right)\right)\left(1-l_{i}(t)-b_{i}(t)\right) \\
& -\alpha l_{i}(t)-\gamma l_{i}(t), \\
\frac{d b_{i}(t)}{d t}= & \gamma l_{i}(t)-\eta b_{i}(t),
\end{aligned}
$$

$1 \leq i \leq N$.

\section{Theoretical Analysis}

System (7) obviously has a unique virus-free equilibrium $E_{0}=$ $(0,0, \ldots, 0)^{T}{ }_{2 N \times 1}$. This section concentrates on the stability of the virus-free equilibrium and persistence of system (7).

First, consider properties of the virus-free equilibrium of system (7). 
Let

$$
\begin{aligned}
\Omega & =\left\{X=\left(x_{1}, x_{2}, \ldots, x_{2 N}\right)^{T} \in \Re_{+}^{2 N} \mid x_{i}+x_{i+N} \leq 1, i\right. \\
& =1,2, \ldots, N\} .
\end{aligned}
$$

Let

$$
x(t)=\left(l_{1}(t), \ldots, l_{N}(t), b_{1}(t), \ldots, b_{N}(t)\right)^{T} .
$$

Then, system (7) can be expressed in the form of a matrix as follows:

$$
\frac{d x(t)}{d t}=W x(t)+Y(x(t)),
$$

with initial condition $x(0) \in \Omega$, where

$$
\begin{aligned}
& W_{2 N \times 2 N}=\left[\begin{array}{cc}
-\alpha I-\gamma I+H & H \\
\gamma I & -\eta I
\end{array}\right], \\
& Y(x(t))_{2 N \times 1}=\left[-\left(l_{1}(t)+b_{1}(t)\right)\right. \\
& \cdot \sum_{j} h_{1 j}\left(l_{j}(t)+b_{j}(t)\right), \ldots,-\left(l_{N}(t)+b_{N}(t)\right) \\
& \cdot \sum_{j} h_{N j}\left(l_{j}(t)+b_{j}(t)\right), \overbrace{0, \ldots, 0}^{N}]^{T} \cdot
\end{aligned}
$$

Let

$$
R_{0}=\frac{\eta(\alpha+\gamma)}{\eta+\gamma} .
$$

Theorem 1. Consider linear system (7):

(a) The virus-free equilibrium $E_{0}=(0,0, \ldots, 0)^{T}{ }_{2 N \times 1}$ is asymptotically stable if $\lambda_{\max }<R_{0}$.

(b) The virus-free equilibrium $E_{0}=(0,0, \ldots, 0)^{T}{ }_{2 N \times 1}$ is unstable if $\lambda_{\max }>R_{0}$.

Proof. The characteristic equation of the Jacobian matrix of system (7) at $E_{0}$ is

$$
\begin{aligned}
\operatorname{det} & (\lambda I-W) \\
& =\operatorname{det}\left(\begin{array}{cc}
(\lambda+\alpha+\gamma) I-H & -H \\
-\gamma I & (\lambda+\eta) I
\end{array}\right)_{2 N \times 2 N} \\
& =\operatorname{det}((\lambda+\alpha+\gamma)(\lambda+\eta) I-((\lambda+\gamma+\eta) H)) \\
& =0 .
\end{aligned}
$$

Equation (14) has two possible cases.

Case $1(\alpha=\eta) \cdot R_{0}=\eta$, and (14) is derived into

$$
(\lambda+\eta+\gamma)^{N} \operatorname{det}((\lambda+\eta) I-H)=0 .
$$

This equation has a negative root $-\eta-\gamma$ with multiplicity $N$; and the remaining $N$ roots of the equation are $\lambda_{k}-\eta$, $1 \leq k \leq N$. If $\lambda_{\max }<R_{0}$, then $\lambda_{k}-\eta \leq \lambda_{\max }-\eta<0$ for all $k$. Hence, all the roots of (14) are negative. So, the virusfree equilibrium of system (7) is asymptotically stable. On the contrary, if $\lambda_{\max }>R_{0}$, then $\lambda_{\max }-\eta>0$. So, (14) has a positive root. As a result, the virus-free equilibrium is unstable.

Case $2(\alpha \neq \eta) .-\eta-\gamma$ is not a root of (14). Thus,

$$
\operatorname{det}\left(\frac{(\lambda+\alpha+\gamma)(\lambda+\eta)}{(\lambda+\gamma+\eta)} I-H\right)=0
$$

This means that $\lambda$ is a root of (14) if and only if $\lambda$ is a root of

$$
\lambda^{2}+a_{k} \lambda+b_{k}=0
$$

where

$$
\begin{aligned}
& a_{k}=\alpha+\gamma+\eta-\lambda_{k}, \\
& b_{k}=(\alpha+\gamma) \eta-\lambda_{k}(\gamma+\eta) .
\end{aligned}
$$

If $\lambda_{\max }<R_{0}$, we have $a_{k}>0$ and $b_{k}>0$. According to the Hurwitz criterion, the two roots of (17) both have negative real parts. So, all roots of (14) have negative real parts. Hence, the virus-free equilibrium is asymptotically stable. Otherwise, if $\lambda_{\max }>R_{0}$, then

$$
\lambda^{2}+a_{k} \lambda+b_{k}=0
$$

has a root with positive real part. As a result, (14) has a root with positive real part. Hence, the virus-free equilibrium is unstable.

The proof is complete.

Then, we consider the global stability of the virus-free equilibrium of system (7).

Lemma 2 (see [31]). Consider a system $d x / d t=f(x)$ that is defined at least in a compact set $C$. Then, $C$ is invariant if, for every point $y$ on $\partial C$, the vector $f(y)$ is tangent to or pointing to $C$.

Lemma 3. The set $\Omega$ is positively invariant for system (7). That is, $x(0) \in \Omega$ implies $x(t) \in \Omega$ for all $t>0$.

Proof. $\partial \Omega$ consists of the following $3 N$ hyperplanes:

$$
\begin{aligned}
S_{i} & =\left\{x \in \Omega \mid x_{i}=0\right\}, \\
T_{i} & =\left\{x \in \Omega \mid x_{i+N}=0\right\}, \\
U_{i} & =\left\{x \in \Omega \mid x_{i}+x_{i+N}=1\right\},
\end{aligned}
$$

which have

$$
\begin{aligned}
\varphi_{i} & =(0, \ldots, 0,-1,0, \ldots, 0) \\
\psi_{i} & =(0, \ldots, 0, \stackrel{i+N}{-1}, 0, \ldots, 0) \\
\xi_{i} & =(0, \ldots, 0, \stackrel{i}{1}, 0, \ldots, 0, \stackrel{i+N}{1}, 0, \ldots, 0)
\end{aligned}
$$


as their respective outer normal vectors. For $1 \leq i \leq N$, we have

$$
\begin{aligned}
\left(\left.\frac{d x}{d t}\right|_{x \in S_{i}} \cdot \varphi_{i}\right) & =-\left(1-x_{i+N}\right) \sum_{j=1}^{N} h_{i j}\left(x_{j}+x_{j+N}\right) \\
& <0, \\
\left(\left.\frac{d x}{d t}\right|_{x \in T_{i}} \cdot \psi_{i}\right) & =-\gamma x_{i}<0, \\
\left(\left.\frac{d x}{d t}\right|_{x \in U_{i}} \cdot \xi_{i}\right) & =-\alpha x_{i}-\eta\left(1-x_{i}\right)<0 .
\end{aligned}
$$

Thus, the claimed result follows from Lemma 2.

Lemma 4 (see [31]). Consider an n-dimensional autonomous system

$$
\frac{d z(t)}{d t}=A z(t)+Q(z(t)), \quad z \in D,
$$

where $A$ is an irreducible $n \times n$ matrix, $D$ is a region containing the origin, $Q(z) \in C$, and $\lim _{x \rightarrow 0}\|Q(z)\| /\|z\|=0$. We assume that there exists a positively invariant compact convex set [16] $C \subset D$ containing the origin, a positive number $r$, and a real eigenvector $\omega$ of $A^{T}$ such that

(C1) $\langle z, \omega\rangle \geq r\|z\|$ for all $z \in C$,

(C2) $\langle Q(z), \omega\rangle \leq 0$ for all $z \in C$,

(C3) the origin forms the largest positively invariant set [16] included in $N=\{z \in C \mid\langle Q(z), \omega\rangle=0\}$.

Then, we have the following:

(1) $\omega<0$ implies that the origin is globally asymptotically stable in $C$.

(2) $\omega>0$ implies that there exists $m>0$ such that, for each $z_{0} \in C-\{0\}$, the solution $\phi\left(t, z_{0}\right)$ to system (7) satisfies $\lim _{t \rightarrow \infty} \inf \left\|\phi\left(t, z_{0}\right)\right\| \geq m$.

Theorem 5. Consider system (7). The virus-free equilibrium $E_{0}=(0,0, \ldots, 0)^{T}{ }_{2 N \times 1}$ is globally asymptotically stable in $\Omega$ if $\lambda_{\text {max }}<R_{0}$.

Proof. Let $C=\Omega, A=W$, and $Q=Y$. As matrix $W^{T}$ is irreducible and all of its nondiagonal entries are nonnegative, from [31] $A^{T}$ has a positive eigenvector $z=\left(z_{1}, z_{2}, \ldots, z_{2 N}\right)$ corresponding to its eigenvalue $\omega$.

Let $r=\min \left\{z_{i}: 1 \leq i \leq 2 N\right\}>0$. Then,

$$
\begin{aligned}
\langle x, z\rangle & \geq r \sum_{i=1}^{2 N} x_{i}=r\|x\|, \\
\langle Q(x), z\rangle & =-\sum_{i=1}^{N} z_{i}\left(x_{i}+x_{i+N}\right) \sum_{j} h_{i j}\left(x_{j}+x_{N+j}\right) \\
& \leq 0 .
\end{aligned}
$$

$\langle Q(x), z\rangle=0$ implies that $x=0$. Hence, the claimed result follows from Lemma 4.

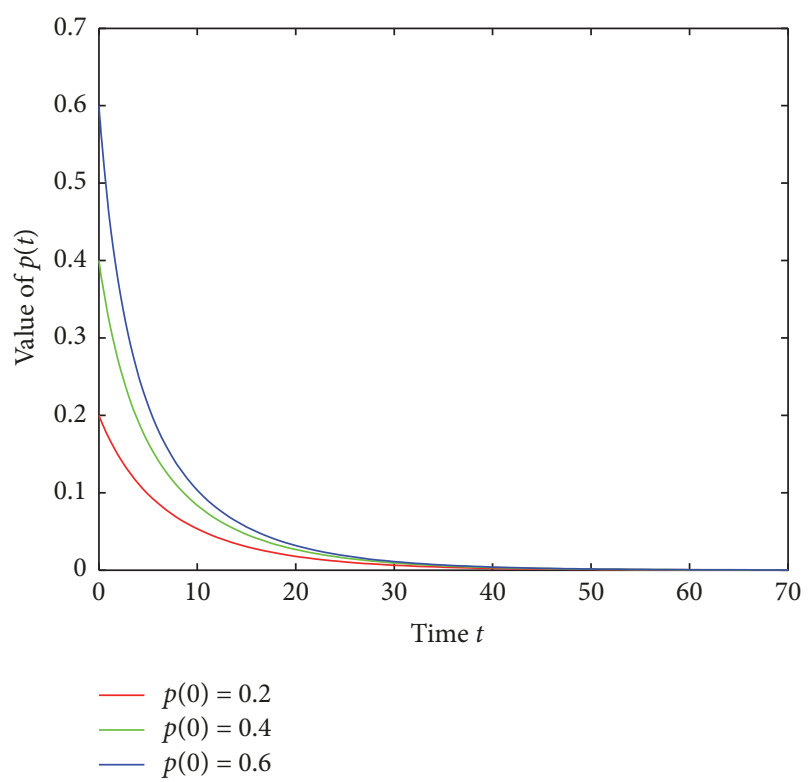

Figure 3: The time plot of $p(t)$ for Example 1 with different initial values.

Second, we investigate the properties of system (7) when $\lambda_{\max }>R_{0}$. From Lemma 4 and Theorem 1, we can easily get the following results.

Theorem 6. Consider linear system (7). If $\lambda_{\max }>R_{0}$, we have

$$
\begin{aligned}
\sum_{i}\left(l_{i}(t)+b_{i}(t)\right)>0 & \Longrightarrow \\
\liminf _{t \rightarrow \infty} \sum_{i}\left(l_{i}(t)+b_{i}(t)\right)>0 . &
\end{aligned}
$$

Remark 7. Theorem 5 shows that the global stability of the virus-free equilibrium implies that the viruses will decline to extinction.

Remark 8. Theorem 6 shows that if $\lambda_{\max }>R_{0}$, then, computer viruses in the network will persist.

\section{Numerical Simulations}

In this section, the main theorems of this paper are verified by some numerical simulations. Let $p(t)$ represent the percentage of infected nodes in all nodes at time $t, p(t)=$ $(1 / N) \sum_{i=1}^{N}\left(l_{i}(t)+b_{i}(t)\right)$.

(1) We consider a two-layer complete graph, which has 250 nodes, and the infection rate of the 1st and the 2nd layer subnetwork is $\beta_{1}=0.0005$ and $\beta_{2}=0.0007$, respectively. Then, $\lambda_{\max }=0.2988$.

Example 1. Based on (1), Figure 3 shows the dynamic behavior of system (7) with $\alpha=0.4, \gamma=0.5$, and $\eta=0.4$ for different initial conditions. By calculation, $R_{0}=0.4$. Because of $\lambda_{\max }<R_{0}$, computer virus would die out.

Example 2. Based on (1), Figure 4 reveals the dynamic behavior of system (7) with $\alpha=0.4, \gamma=0.5$, and $\eta=0.12$ 


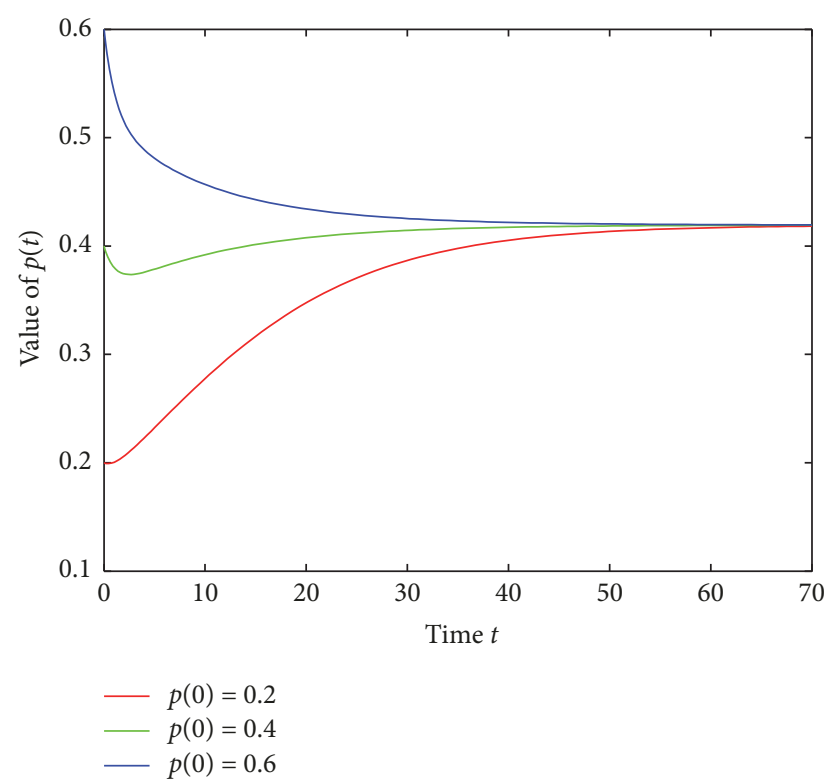

Figure 4: The time plot of $p(t)$ for Example 2 with different initial values.

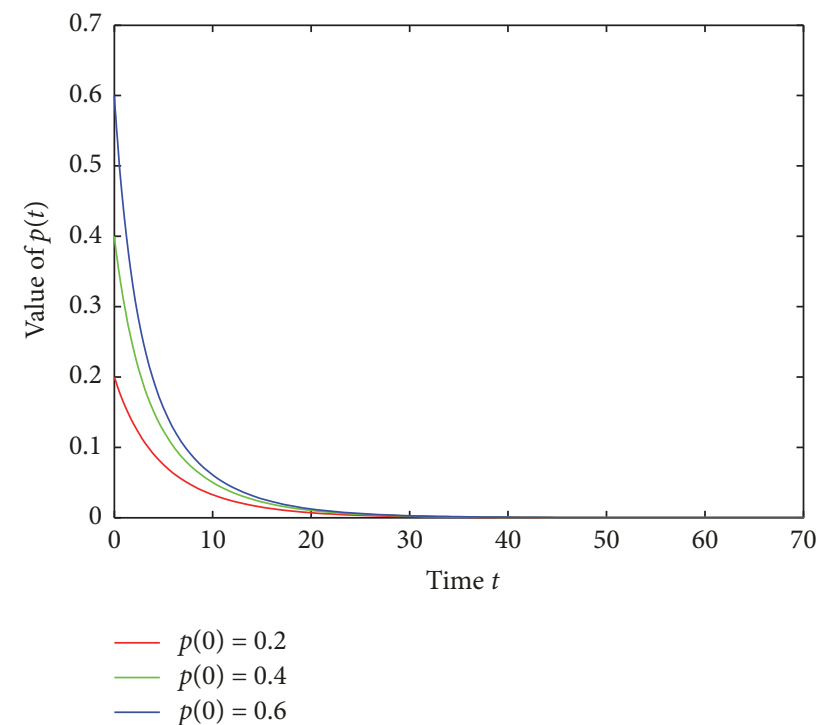

FIgURE 5: The time plot of $p(t)$ for Example 3 with different initial values.

in different initial conditions. Then $R_{0}=0.1742$. Because of $\lambda_{\text {max }}>R_{0}$, computer virus would persist.

(2) We take a three-layer Erdos-Renyi random graph, which has 250 nodes, with random connection probability 0.8 . The infection rate of the 1 st, 2 nd, and 3 rd layer subnetwork is $\beta_{1}=0.0005, \beta_{2}=0.0006$, and $\beta_{3}=0.0007$, respectively. Then, $\lambda_{\max }=0.3583$.

Example 3. Based on (2), Figure 5 illustrates the dynamic behavior of system (7) with $\alpha=0.4, \gamma=0.5$, and $\eta=0.6$ for different initial conditions. By calculation, $R_{0}=0.4909$. Because of $\lambda_{\max }<R_{0}$, computer virus would die out.

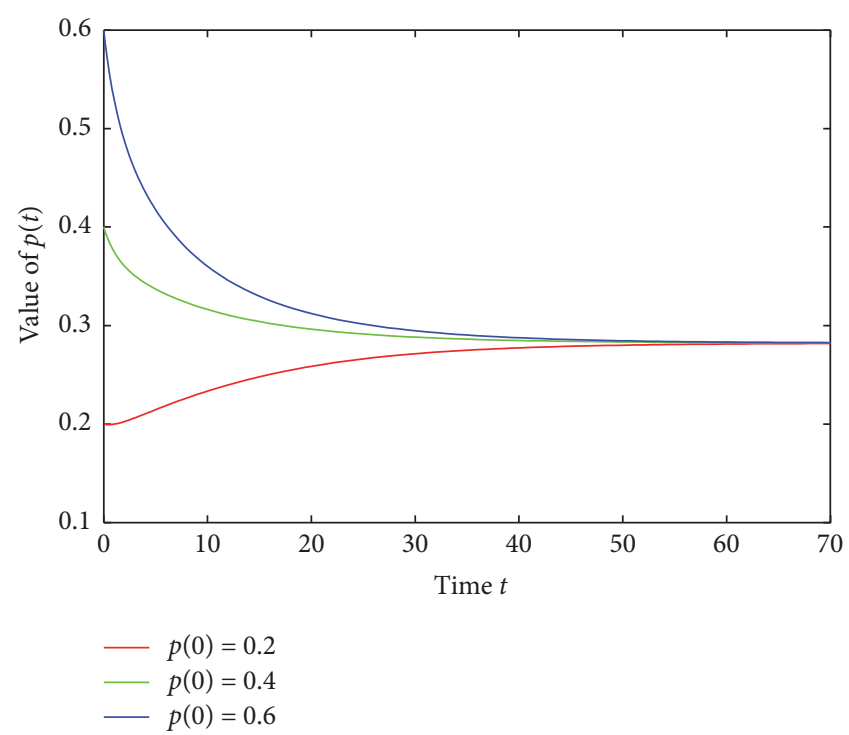

Figure 6: The time plot of $p(t)$ for Example 4 with different initial values.

Example 4. Based on (2), Figure 6 shows the dynamic behavior of system (7) with $\alpha=0.4, \gamma=0.5$, and $\eta=0.2$ in different initial conditions. Then $R_{0}=0.2571$. Because of $\lambda_{\max }>R_{0}$, computer virus would persist.

(3) We take a two-layer Barabási-Albert (BA) scale-free graph, which has 250 nodes. The infection rate of the 1st and 2nd layer subnetwork is $\beta_{1}=0.02$ and $\beta_{2}=0.03$, respectively. Then, $\lambda_{\max }=0.2946$.

Example 5. Based on (3), Figure 7 illustrates the dynamic behavior of system (7) with $\alpha=0.4, \gamma=0.5$, and $\eta=0.6$ for different initial conditions. By calculation, $R_{0}=0.4909$. Because of $\lambda_{\max }<R_{0}$, computer virus would die out.

Example 6. Based on (3), Figure 8 shows the dynamic behavior of system (7) with $\alpha=0.4, \gamma=0.5$, and $\eta=0.1$ in different initial conditions. Then $R_{0}=0.15$. Because of $\lambda_{\text {max }}>R_{0}$, computer virus would persist.

(4) Consider a multilayer network consisting of three subnetworks; each subnetwork has 250 nodes. The subnetwork of the 1st, 2nd, and 3rd is complete connected network, random network, and scale-free network, respectively. And the connection probability of the random subnetworks is 0.7 . Besides, the infection rate of the 1st, 2 nd, and 3 rd subnetwork is $\beta_{1}=0.0002, \beta_{2}=0.0003$, and $\beta_{3}=0.001$, respectively. Then, $\lambda_{\text {max }}=0.2760$.

Example 7. Based on (4), Figure 9 illustrates the dynamic behavior of system (7) with $\alpha=0.4, \gamma=0.5$, and $\eta=0.6$ for different initial conditions. By calculation, $R_{0}=0.4909$. Because of $\lambda_{\max }<R_{0}$, computer virus would die out.

Example 8. Based on (4), Figure 10 shows the dynamic behavior of system (7) with $\alpha=0.1, \gamma=0.5$, and $\eta=0.1$ in 


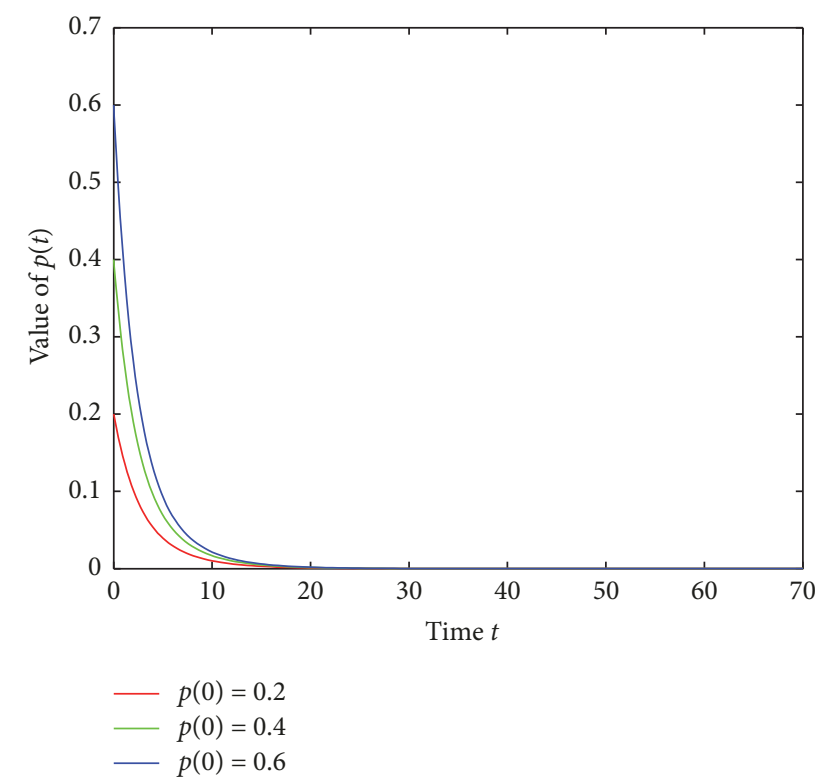

Figure 7: The time plot of $p(t)$ for Example 5 with different initial values.

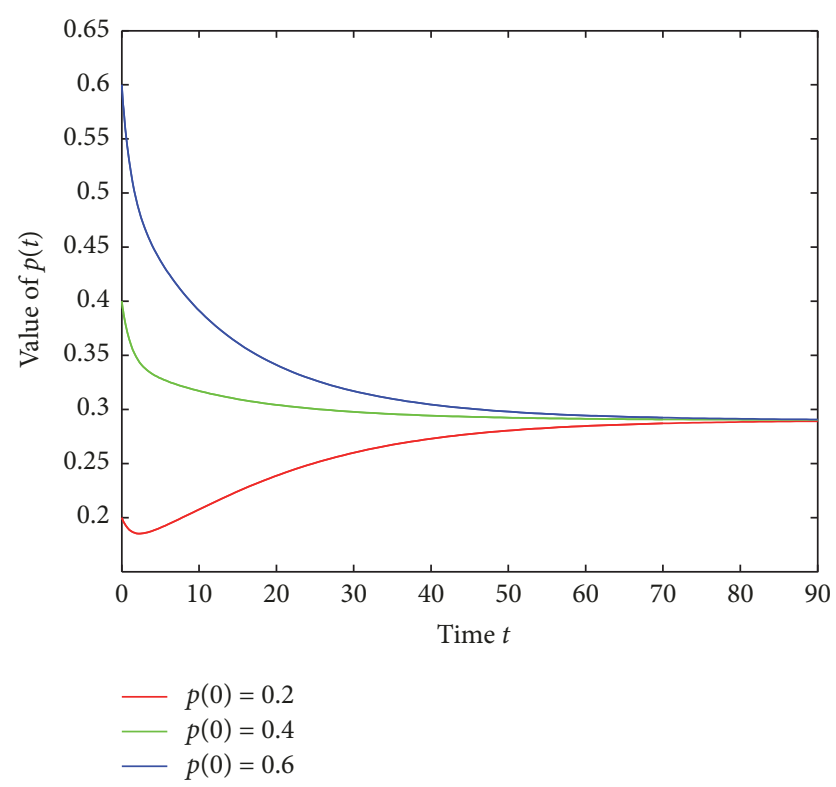

Figure 8: The time plot of $p(t)$ for Example 6 with different initial values.

different initial conditions. Then $R_{0}=0.1$. Because of $\lambda_{\max }>$ $R_{0}$, computer virus would persist.

(5) The propagation threshold $R_{0}$ plays a vital role in determining the dynamics of system (7). As $\partial R_{0} / \partial \alpha>0$, $\partial R_{0} / \partial \eta>0, R_{0}$ is strictly increasing with respect to the parameters $\alpha, \eta$ (see Figure 11). As $\partial R_{0} / \partial \gamma=\eta(\eta-\alpha) /(\eta+\gamma)^{2}$, when $\eta>\alpha, R_{0}$ increases as $\gamma$ increases; when $\eta<\alpha, R_{0}$ decreases as $\gamma$ increases (see Figure 12).

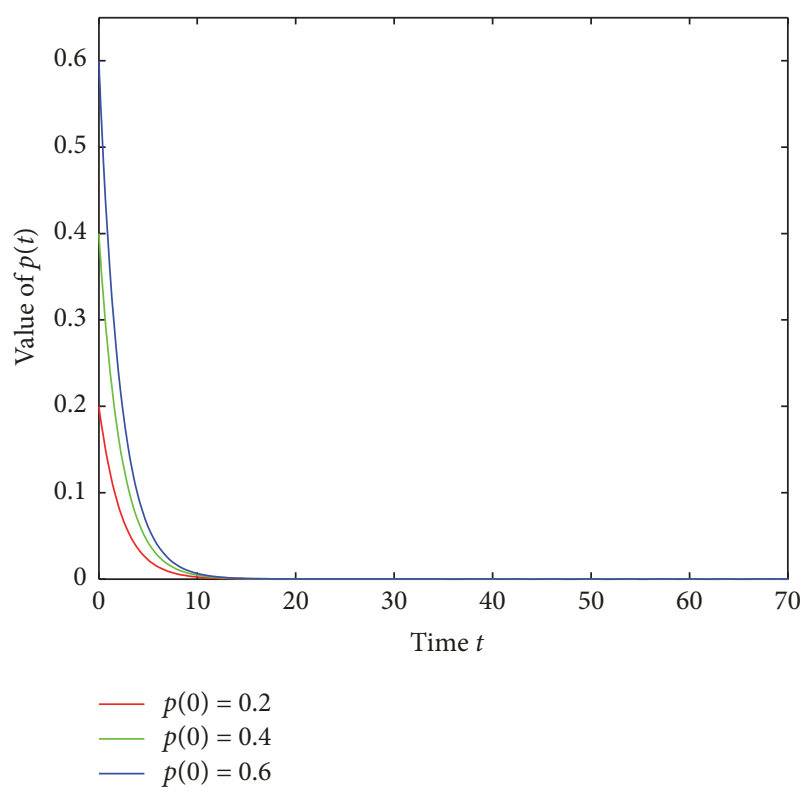

FIGURE 9: The time plot of $p(t)$ for Example 7 with different initial values.

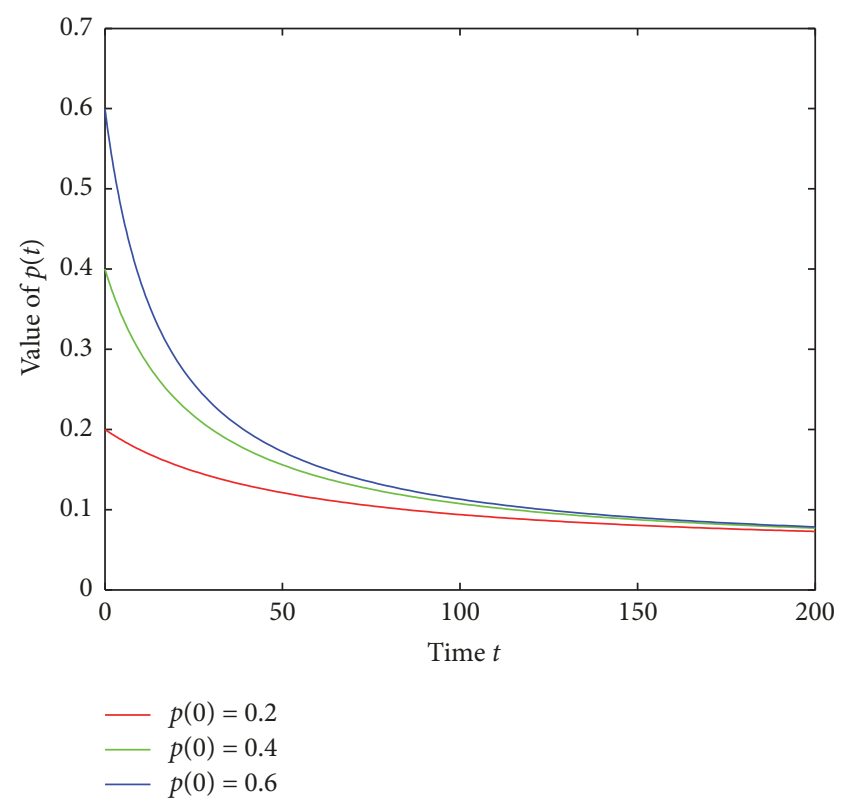

Figure 10: The time plot of $p(t)$ for Example 8 with different initial values.

\section{Conclusions}

To explore the propagation mechanism of computer viruses on multilayer network, a novel computer virus propagation model has been proposed. The theoretical analysis of model exhibited that computer viral prevalence is deeply determined by the maximum eigenvalue of the multilayer networks. Then, the global stability of virus-free equilibrium and the persistence of computer virus propagation have been proved. Some numerical simulations have also been given. 


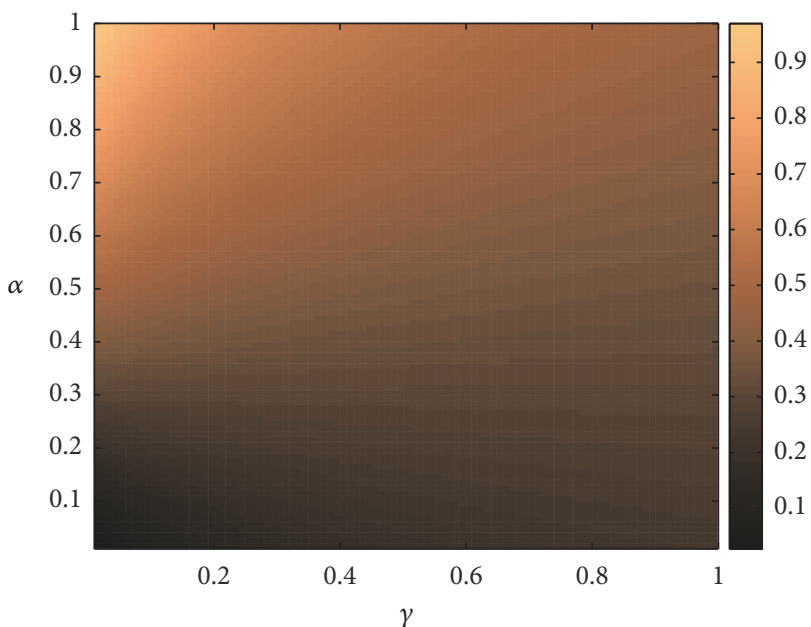

FIgURE 11: Values of $R_{0}$ as a function of varying $\alpha$ and $\gamma$ with fixing the parameter $\eta=0.3$.

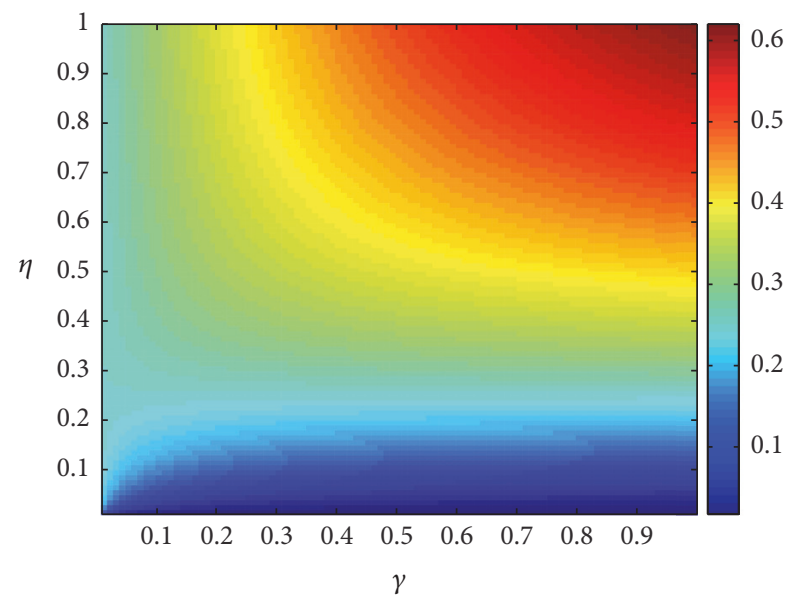

FIGURE 12: Values of $R_{0}$ as a function of varying $\eta$ and $\gamma$ with fixing the parameter $\alpha=0.25$.

As it is well known, the topology of the Internet is varying constantly rather than being static. A dynamic switching network is a network whose links will change (dissipate and emerge) and then the structure of the networks will be different in the distinct time. Therefore, it is of practical importance to understand the effect of dynamic switching networks on virus spreading. Our next work is to study the spreading behavior of computer viruses on dynamic switching networks.

\section{Conflicts of Interest}

The author declares that they have no conflicts of interest.

\section{Acknowledgments}

This work is supported by Natural Science Foundation of Guangdong Province, China (no. 2014A030310239).

\section{References}

[1] F. Cohen, "Computer viruses: theory and experiments," Computers \& Security, vol. 6, no. 1, pp. 22-35, 1987.

[2] J. Kephart and S. White, "Directed-graph epidemiological models of computer viruses," in Proceedings of the 1991 IEEE Computer Society Symposium on Research in Security and Privacy, pp. 343-359, Oakland, CA, USA, 1991.

[3] J. Ren, X. Yang, Q. Zhu, L.-X. Yang, and C. Zhang, "A novel computer virus model and its dynamics," Nonlinear Analysis: Real World Applications, vol. 13, no. 1, pp. 376-384, 2012.

[4] Q. Zhu, X. Yang, and J. Ren, "Modeling and analysis of the spread of computer virus," Communications in Nonlinear Science and Numerical Simulation, vol. 17, no. 12, pp. 5117-5124, 2012.

[5] C. Gan and X. Yang, “Theoretical and experimental analysis of the impacts of removable storage media and antivirus software on viral spread," Communications in Nonlinear Science and Numerical Simulation, vol. 22, no. 1-3, pp. 167-174, 2015.

[6] B. K. Mishra and S. K. Pandey, "Dynamic model of worms with vertical transmission in computer network," Applied Mathematics and Computation, vol. 217, no. 21, pp. 8438-8446, 2011.

[7] L.-X. Yang, X. Yang, and Y. Wu, “The impact of patch forwarding on the prevalence of computer virus: A theoretical assessment approach," Applied Mathematical Modelling, vol. 43, pp. 110-125, 2017.

[8] C. Gan, X. Yang, Q. Zhu, J. Jin, and L. He, "The spread of computer virus under the effect of external computers," Nonlinear Dynamics, vol. 73, no. 3, pp. 1615-1620, 2013.

[9] L.-X. Yang, X. Yang, L. Wen, and J. Liu, "A novel computer virus propagation model and its dynamics," International Journal of Computer Mathematics, vol. 89, no. 17, pp. 2307-2314, 2012.

[10] P. Van Mieghem, J. Omic, and R. Kooij, "Virus spread in networks," IEEE/ACM Transactions on Networking, vol. 17, no. 1, pp. $1-14,2009$.

[11] M. Karsai, M. Kivelä, R. K. Pan et al., "Small but slow world: How network topology and burstiness slow down spreading," Physical Review E: Statistical, Nonlinear, and Soft Matter Physics, vol. 83, no. 2, Article ID 025102, 2011.

[12] J. C. Wierman and D. J. Marchette, "Modeling computer virus prevalence with a susceptible-infected-susceptible model with reintroduction," Computational Statistics \& Data Analysis, vol. 45, no. 1, pp. 3-23, 2004.

[13] C. Griffin and R. Brooks, "A note on the spread of worms in scale-free networks," IEEE Transactions on Systems, Man, and Cybernetics, Part B: Cybernetics, vol. 36, no. 1, pp. 198-202, 2006.

[14] L.-X. Yang, M. Draief, and X. Yang, "The impact of the network topology on the viral prevalence: a node-based approach," PLoS ONE, vol. 10, no. 7, article e0134507, 2015.

[15] T. Zhou, J.-G. Liu, W.-J. Bai, G. Chen, and B.-H. Wang, "Behaviors of susceptible-infected epidemics on scale-free networks with identical infectivity," Physical Review E: Statistical, Nonlinear, and Soft Matter Physics, vol. 74, no. 5, Article ID 056109, 2006.

[16] https://en.wikipedia.org/wiki/Positive_invariant_set.

[17] H. J. Shi, Z. S. Duan, and G. R. Chen, "An SIS model with infective medium on complex networks," Physica A: Statistical Mechanics and its Applications, vol. 387, no. 8-9, pp. 2133-2144, 2008.

[18] L. Wen and J. Zhong, "Global asymptotic stability and a property of the SIS model on bipartite networks," Nonlinear Analysis: Real World Applications, vol. 13, no. 2, pp. 967-976, 2012. 
[19] A. d'Onofrio, "A note on the global behaviour of the networkbased SIS epidemic model," Nonlinear Analysis: Real World Applications, vol. 9, no. 4, pp. 1567-1572, 2008.

[20] C. Castellano and R. Pastor-Satorras, "Thresholds for epidemic spreading in networks," Physical Review Letters, vol. 105, no. 21, Article ID 218701, 2010.

[21] L. C. Chen and K. M. Carley, "The impact of countermeasure propagation on the prevalence of computer viruses," IEEE Transactions on Systems, Man, and Cybernetics, Part B: Cybernetics, vol. 34, no. 2, pp. 823-833, 2004.

[22] M. Draief, A. Ganesh, and L. Massouilié, "Thresholds for virus spread on networks," The Annals of Applied Probability, vol. 18, no. 2, pp. 359-378, 2008.

[23] Y. Moreno, R. Pastor-Satorras, and A. Vespignani, "Epidemic outbreaks in complex heterogeneous networks," The European Physical Journal B, vol. 26, no. 4, pp. 521-529, 2002.

[24] C. Gan, "Modeling and analysis of the effect of network eigenvalue on viral spread," Nonlinear Dynamics, vol. 84, no. 3, pp. 1727-1733, 2016.

[25] P. Van Mieghem and E. Cator, "Epidemics in networks with nodal self-infection and the epidemic threshold," Physical Review E: Statistical, Nonlinear, and Soft Matter Physics, vol. 86, no. 1, Article ID 016116, 2012.

[26] Y. Wang, D. Chakrabarti, C. Wang, and C. Faloutsos, "Epidemic spreading in real networks: an eigenvalue viewpoint," in Proceedings of the 22nd International Symposium on Reliable Distributed Systems (SRDS '03), pp. 25-34, Florence, Italy, October 2003.

[27] M. Youssef and C. Scoglio, "An individual-based approach to SIR epidemics in contact networks," Journal of Theoretical Biology, vol. 283, pp. 136-144, 2011.

[28] R. Pastor-Satorras and A. Vespignani, "Epidemic spreading in scale-free networks," Physical Review Letters, vol. 86, no. 14, pp. 3200-3203, 2001.

[29] R. Pastor-Satorras and A. Vespignani, "Epidemic dynamics and endemic states in complex networks," Physical Review E: Statistical, Nonlinear, and Soft Matter Physics, vol. 63, no. 6, Article ID 066117, 2001.

[30] M. Barthélemy, A. Barrat, R. Pastor-Satorras, and A. Vespignani, "Velocity and hierarchical spread of epidemic outbreaks in scale-free networks," Physical Review Letters, vol. 92, no. 17, Article ID 178701, 2004.

[31] A. Lajmanovich and J. A. Yorke, "A deterministic model for gonorrhea in a nonhomogeneous population," Mathematical Biosciences, vol. 28, no. 3/4, pp. 221-236, 1976. 


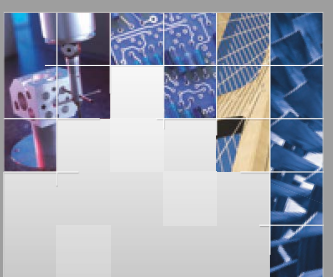

\section{Enfincering}
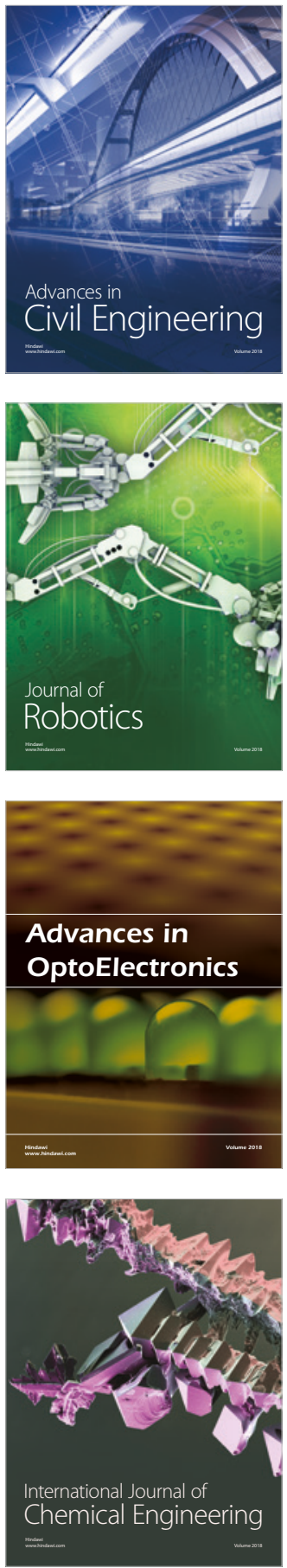

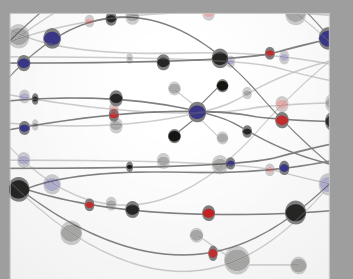

\section{Rotating \\ Machinery}

The Scientific World Journal

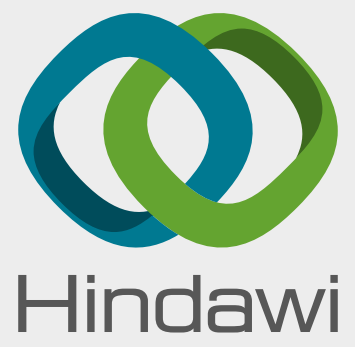

Submit your manuscripts at

www.hindawi.com
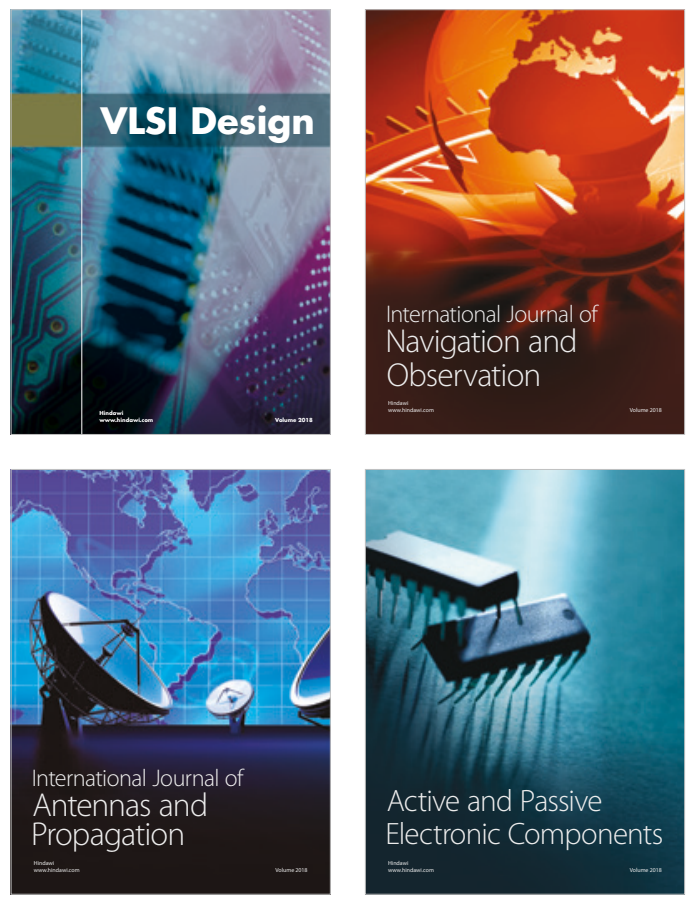
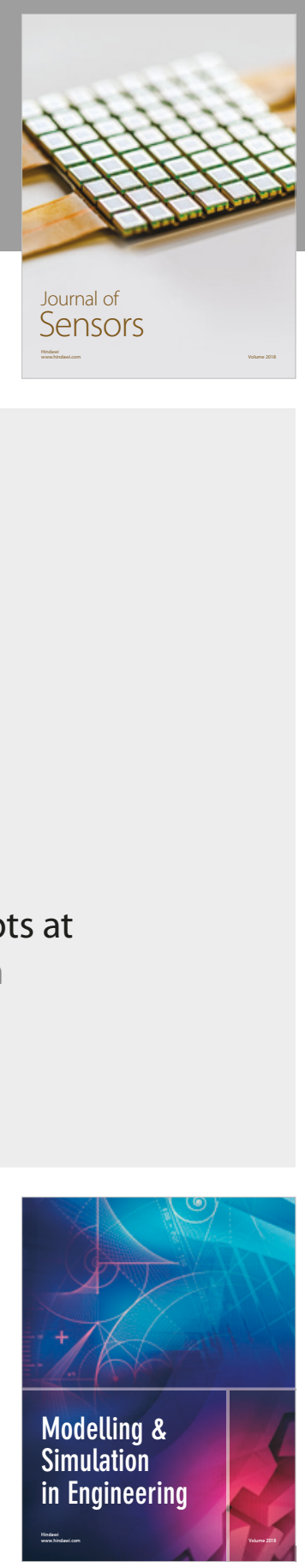

\section{Advances \\ Multimedia}
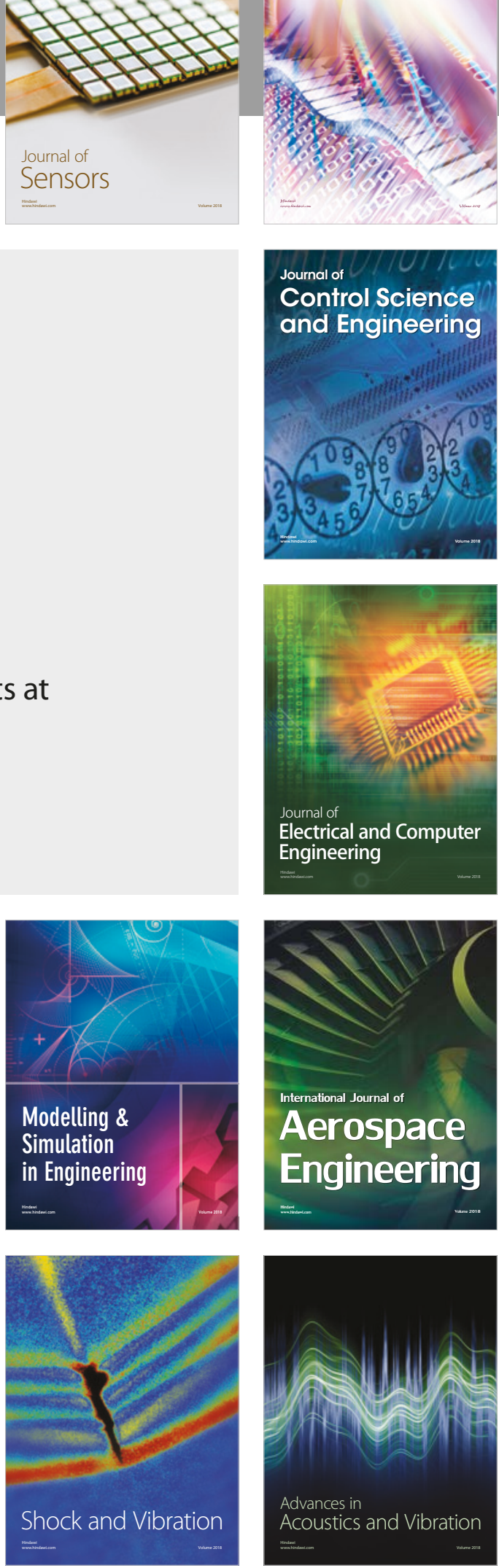